\title{
Excellent Functionality Despite Clinico-Radiological Deformity in Osteomyelitis Variolosa - A Case Report
}

\author{
Aakash Mugalur, Sunil M. Shahane, Ashwin Samant, Aditya C. Pathak* and Rajeev Reddy
}

Department of Orthopaedics, $R$ N Cooper Hospital, Vile Parle (W), Mumbai, India

\begin{abstract}
Despite the eradication of smallpox from the world in 1980 the osteo-articular sequelae of smallpox are still occasionally noticed in previously endemic areas. The sequelae of osteomyelitis variolosa may raise a diagnostic challenge to the untrained eyes of the surgeon. We present a case of "osteomyelitis variolosa" in a 70 years old patient. The patient had bilateral dislocation of the elbow joint with multidirectional instability. There was distortion of the articular surfaces and ankylosis of the bilateral proximal radio-ulnar joint. Hypoplasia of the right ulna with short fourth and fifth metacarpals of the left hand and hypoplasia of right fourth metacarpal with cortical thickening was noted radiologically. The patient had minimal disability of his elbows despite the striking radiological abnormality and was functionally independent.
\end{abstract}

Keywords: Bilateral dislocation, disability, elbow, osteomyelitis variolosa, smallpox.

\section{INTRODUCTION}

World Health Organisation declared the world free of Smallpox in the year 1980 making it the earliest of infective diseases to be eradicated from the world [1]. Smallpox was an infective disease caused by Variola virus, of the genus Orthopoxvirus. The term osteomyelitis variolosa refers to the osteo-articular affection of Variola major, only seen in cases arising in childhood and tends to affect predominantly the elbow joints among other joints $[2,3]$.

The orthopaedic manifestations and pathophysiology of the disease were described in 1958 and 1959 by Cockshott \& McGregor [2, 3]. In the acute cases of osteomyelitis elementary bodies of the virus were present in the fluid from affected joints which showed no growth on culture for pyogenic organisms. It differed from acute bacterial osteomyelitis showing no response to antibiotics and chemotherapy. The natural course of the disease was unaffected by the antibiotic therapy and the radiological changes were characteristic of smallpox [4]. Though acute cases of osteomyelitis variolosa are not seen since the eradication of smallpox, the sequelae of the disease are still occasionally noticed in previously endemic areas. Orthopaedic manifestations of the disease are largely forgotten and hence can raise a diagnostic challenge to the untrained eye. We present a case of osteomyelitis variolosa predominantly affecting the elbow joints, in a 70 years old patient who sought orthopaedic help for bilateral knee pain and back pain.

\section{CASE REPORT}

A 70 years old patient sought orthopaedic care due to progressively increasing pain in the bilateral knee joints and

*Address correspondence to this author at the Department of Orthopaedics, R N Cooper Hospital, Vile Parle( W), Mumbai, India;

Tel: +91-9869183922,+91-9819197632; E-mail: acp.ortho@gmail.com difficulty to sit cross-legged and squat since the past three months. The patient also complained of low back pain with no radicular symptoms. The pain was insidious in onset with no history of trauma. The patient had right ankle pain and difficulty in range of motion the symptoms of which started at the age of 48 years. The right ankle joint was subjected to arthrodesis by an orthopaedic surgeon at the age of 50 years. On examination the patient had signs and symptoms suggestive of bilateral knee osteoarthritis and lumbar canal stenosis. The ankle examination revealed the healed surgical scar on the anterolateral aspect of the right ankle joint. Clinically the ankle joint was arthrodesed with no residual tenderness. The patient had features suggestive of severe tricompartmental osteoarthritis in bilateral knee joints but the degenerative changes and osteophytes were more significant on the left side. The radiographs of the right ankle confirmed sound bony ankylosis (Fig. 1). Spine radiographs and MRI scan revealed lumbar degenerative left sided scoliosis, peridiscal osteophytes, endplate degenerative changes, facet joint arthropathy, lumbar canal stenosis from L3 to S1 levels and degenerative cervical spine with $\mathrm{C} 3-\mathrm{C} 7$ posterior disc protrusion compromising the spinal canal but no neurological compression. Bilateral hip and sacroiliac joints were normal on MRI screening.

The patient also had conspicuous bilateral elbow deformities but he had no functional limitations due to elbow affection. The patient had a history of fever with rash at the age of eight years. The patient had affection of bilateral elbow joints during the febrile episode in the form of pain and swelling and no treatment was taken. There was no history of trauma, osteoarticular tuberculosis, or any history suggestive of congenital affection. The patient did not get vaccinated to the best of his knowledge. Currently, on clinical examination the bilateral elbow joints were dislocated postero-medially with prominence of distal end of the humerus laterally and that of the proximal radio-ulnar complex medially. There was no joint line tenderness. 


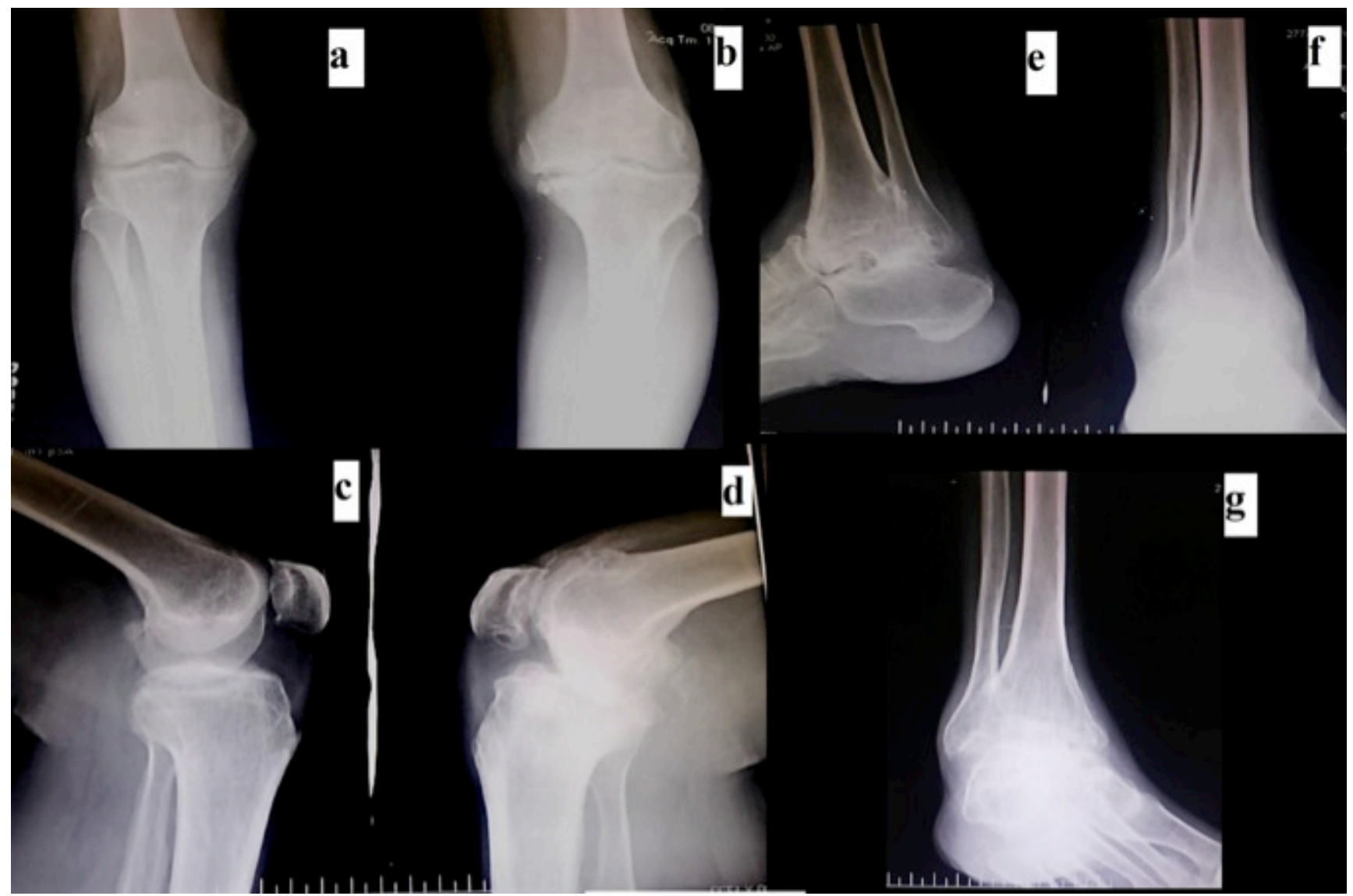

Fig. (1). Knee and ankle radiographs. a \& c) Anteroposterior and lateral views of the right knee joint showing changes of osteoarthritis. b \& d) Anteroposterior and lateral views of the left knee joint showing changes of osteoarthritis. The degenerative changes are more profound. e, f, g) Lateral, anteroposterior and mortise views of the right ankle showing bony ankylosis.

Healed puckered scars were presented on the posteromedial aspect of bilateral elbows. There was no ulnar nerve thickening. Wasting of muscles could not be commented upon due to bilateral affection. The fourth and fifth ray were shortened on the left hand. On the right side the arc of motion was 140 degrees from 30 degree of hyperextension to 110 degree of flexion. On the left side, there was flexion deformity of 30 degree with further flexion up to 110 degree. The pronation was restricted to 20 degree on the right side and 30 degree on the left side with the full range supination bilaterally up to 90 degree (Figs. 2, 3). All movements were pain-free throughout the possible range of motion. There was no distal neurovascular deficit. The bilateral shoulder, wrist and hand joints were normal on clinical examination. On clinical examination of the face, we observed deep pitted scars anterior to the pinna which could be due to the healing of the smallpox lesions. On radiographs bilateral posteromedial dislocation of elbow joints was observed. The articular surfaces were deformed and incongruent. There was fusion of the proximal radius and ulna bilaterally. Heterotopic calcification was observed in bilateral elbow joints. There was marked hypoplasia of the right ulna and short fourth and fifth metacarpals were observed in the left hand. The patient also had hypoplasia of right fourth metacarpal with cortical thickening (Fig. 4). The complete hemogram, erythrocyte sedimentation rate, serum levels of calcium, phosphorus, alkaline phosphatase \& uric acid were within normal limits. Rheumatoid factor was negative. Screening renal and liver function tests were normal.

The patient was right hand dominant and was an automobile mechanic by occupation. Despite the striking clinical deformity and the radiological abnormalities, the patient had no complaints with respect to the bilateral elbow joints. The patient had minimal disability of the elbows despite the striking radiological appearance and was functionally independent. The patient was an automobile mechanic by occupation and was able to lift heavy weights until retirement from occupation.

\section{DISCUSSION}

"Osteomyelitis variolosa" is a well-known viral infection of the bone and is often described as destructive, unpreventable and untreatable [5]. The World Health Organisation declared "Smallpox free - world" in the year 1980 [1]. Three decades have passed by since the eradication of the disease. Acute cases of osteomyelitis variolosa are not seen since the eradication. But none the less sequelae of the disease are still occasionally noticed in previously endemic areas especially after a recent trauma [6-8]. Studies by Cockshott et al. concluded that during an epidemic of smallpox, up to $0.5 \%$ of all patients and especially 2 to $5 \%$ of affected children could have long term osteoarticular sequelae $[2,3]$. Though any bone or joint can be involved in 


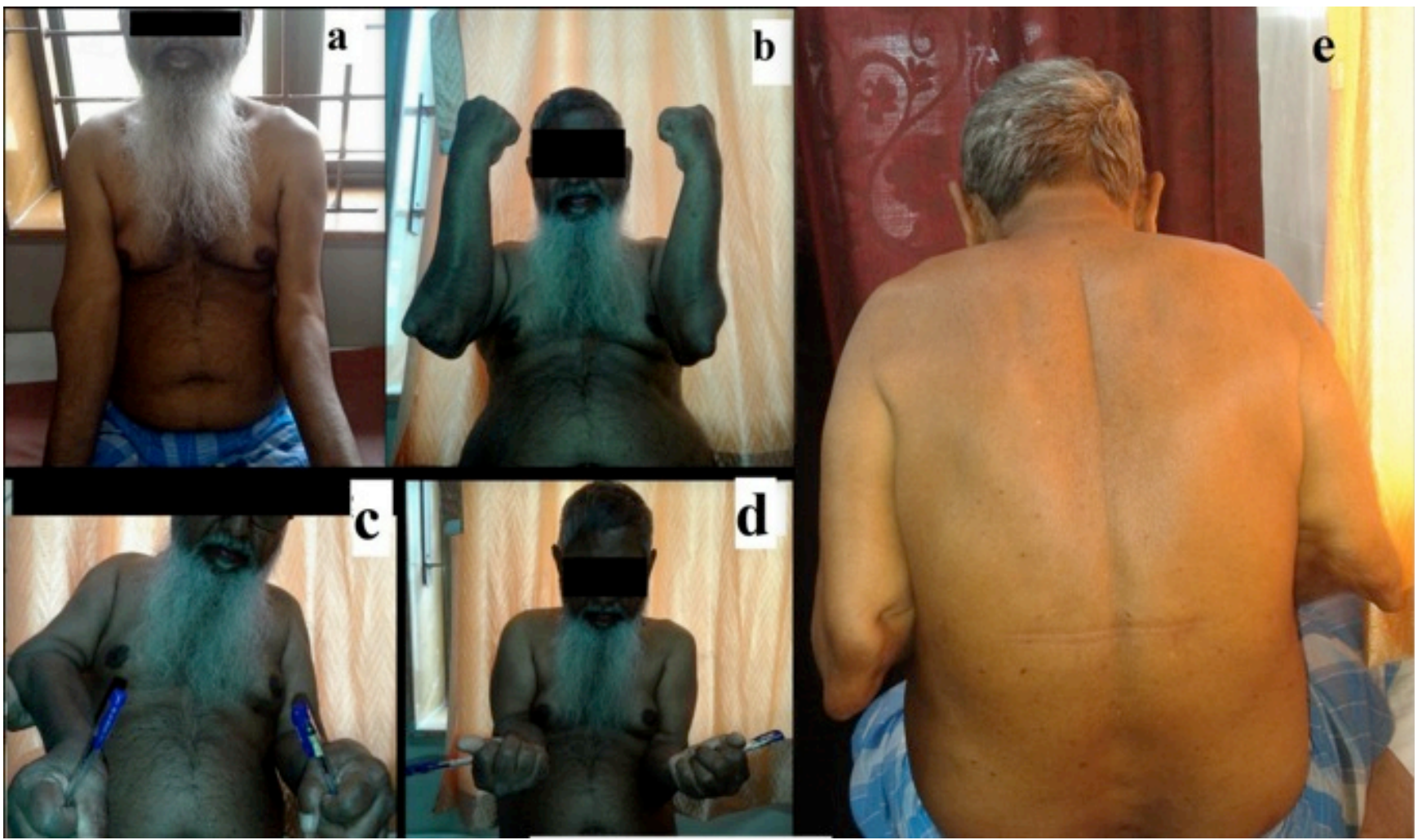

Fig. (2). Clinical photographs. a) Bilateral elbow joints as viewed anteriorly. b) Bilateral elbow joints with maximal flexion. The prominence of distal humerus and the proximal radio-ulnar complex can be noted on lateral and medial sides respectively. c) Limitation of pronation movement bilaterally. d) Near full range of supination. e) Puckered scars reminiscent of healed sinuses on the posteromedial aspect of elbow.

osteomyelitis variolosa, elbow joints are involved in about $80 \%$ of the cases, hands and wrists in $20 \%$, ankles and feet in $13 \%$. Variolar involvement of the knees, hips, shoulders, skull, spine, thorax, or pelvis is considered to be rare $[2,3]$. Though the exact reason behind involvement of the elbow joint is not known, one of the hypotheses is that high stresses in the elbow joint predispose it to localisation during the viraemia phase [2,3]. Pathological foci in bones lead to destruction of the perimetaphyseal structures, epiphyseal separation and to bony changes in diaphysis [9]. The pathomechanism of joint destruction is attributed to the transverse metaphysitis [10], leading to the destruction and displacement of the epiphysis. Thereafter the displaced fragments were either resorbed or were in some cases extruded through the sinuses. Subsequent deformities could be the result of reparative ossification and distortion, cessation of the longitudinal growth of bones, probably due to destruction of the physeal growth plate [11].

In adults in the post eradication era the presenting complaints could be deformities, arthritis or instability. The spectrum of skeletal involvement is wide and varied. The bones may be irregular, sclerosed or thickened with disturbance in the longitudinal growth. The articular surfaces may be deformed leading to precocious osteoarthritis, the joints could be flail with subluxation of the articular surfaces or frank dislocation, or may present with frank ankylosis [6]. Elbow joints normally ended in ankylosis and ankle involvement resulted in hypermobility [4]. Bilateral affection of joints is a conspicuous feature of osteomyelitis variolosa and elbow joints are the most commonly affected joints. But it can affect any joint but affects almost exclusively the appendicular skeleton.

Balaji [7] reported one such case in a 70-years-old woman with hypoplastic lateral femoral condyle of the right knee, patella baja, ankylosed right elbow, dislocated left elbow with multidirectional instability, short third and fourth metacarpals of the left hand, bilateral valgus deformity of ankle with destruction of talus. Arora et al. [6] \& Singh [11] reported successful surgical treatment of distal humerus fracture in osteomyelitis variolosa. They concluded that the fractured bones tend to heal normally despite distortion of the medullary structure.

Our patient was treated by ankle arthrodesis for painful and symptomatic right ankle joint affection at a relatively young age. This could be suggestive of precocious ankle joint arthritis [4] secondary to osteomyelitis. However in the absence of details of the surgery and preoperative radiographs, it is difficult to comment whether ankle arthritis was secondary to variolar affection of the ankle which developed degenerative changes over the period of time. However, there was no history of trauma to the ankle or any neurological deficit in the lower limbs. But provided that it was secondary to the osteomyelitis variolosa, we can infer ankylosis is sound in cases of patients affected by osteomyelitis variolosa.

Though a proper history, appropriate clinical examination and radiology almost always help us to establish a diagnosis, we need to be aware of the some of the differential diagnoses. We need to rule out traumatic dislocation. 


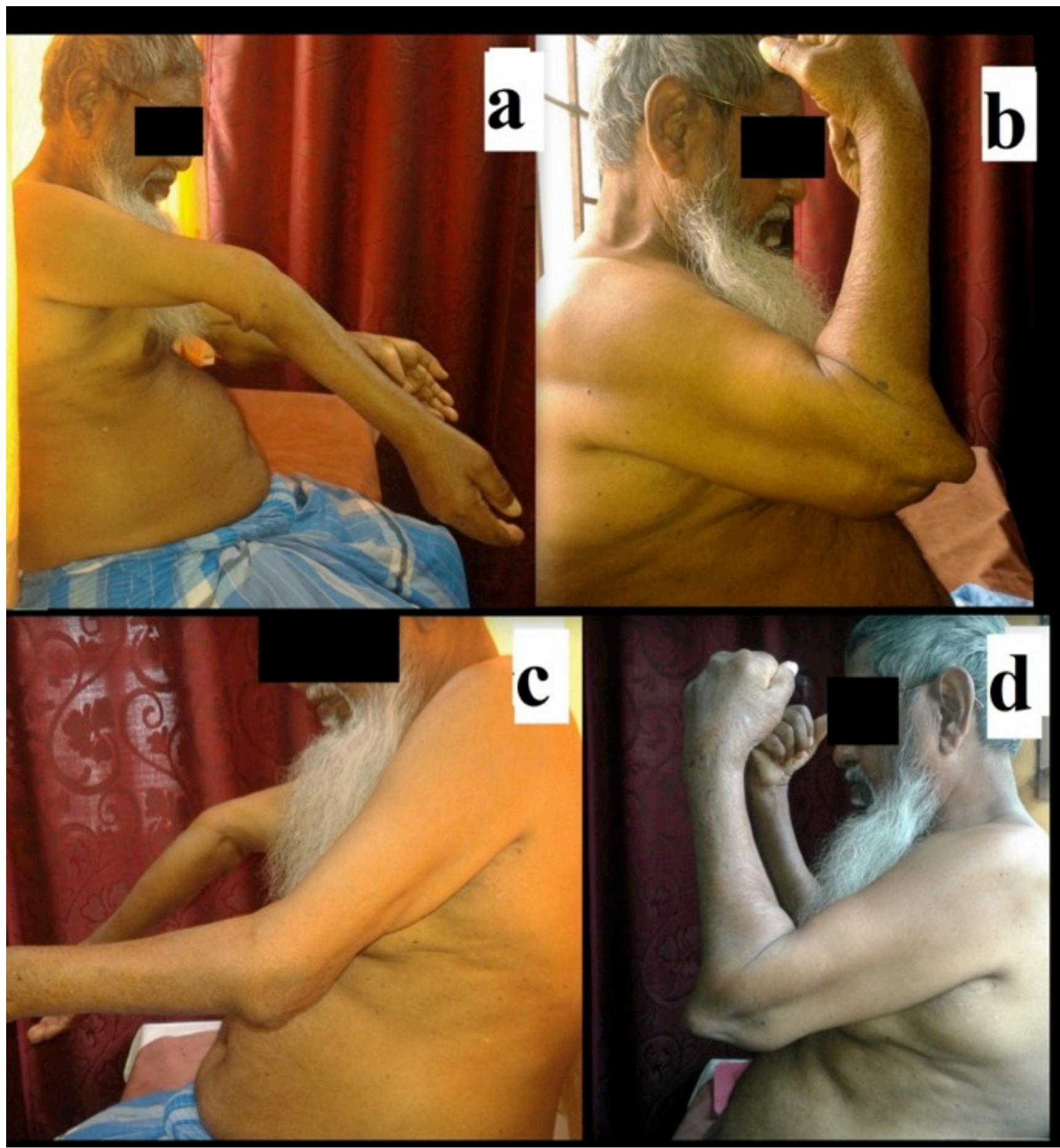

Fig. (3). Range of movement of elbow. a, b) Maximal extension and flexion of right elbow as noted in profile. Note hyperextension on extension view and prominence of distal end of humerus on flexion view. c, d) Maximal extension and flexion of left elbow as noted in profile.

Traumatic bilateral elbow dislocation is rare except in gymnasts [12]. Pyogenic arthritis is one of the differential diagnoses but bilateral involvement of the elbow with involvement of humerus, radius and ulna bilaterally is rare [8]. Bilateral elbow dislocation can also be observed in Larsen syndrome. But multiple congenital joint dislocations and facial abnormalities characteristic of Larsen syndrome are absent in smallpox. Absence of joint deformities before the onset of fever with rash and the lack of typical facial profile rules out Larsen syndrome [13]. Moreover, in smallpox the possible growth disorders have a varied layout. Other differential diagnoses include Achondroplasia [14], pseudohypothyroidism [14], sequelae of chronic burns at hands level [8,9], Leprosy [8] and congenital dysplasias [8]. Most of them can be ruled out through a proper detailed history and clinico-radiological co-relation. 


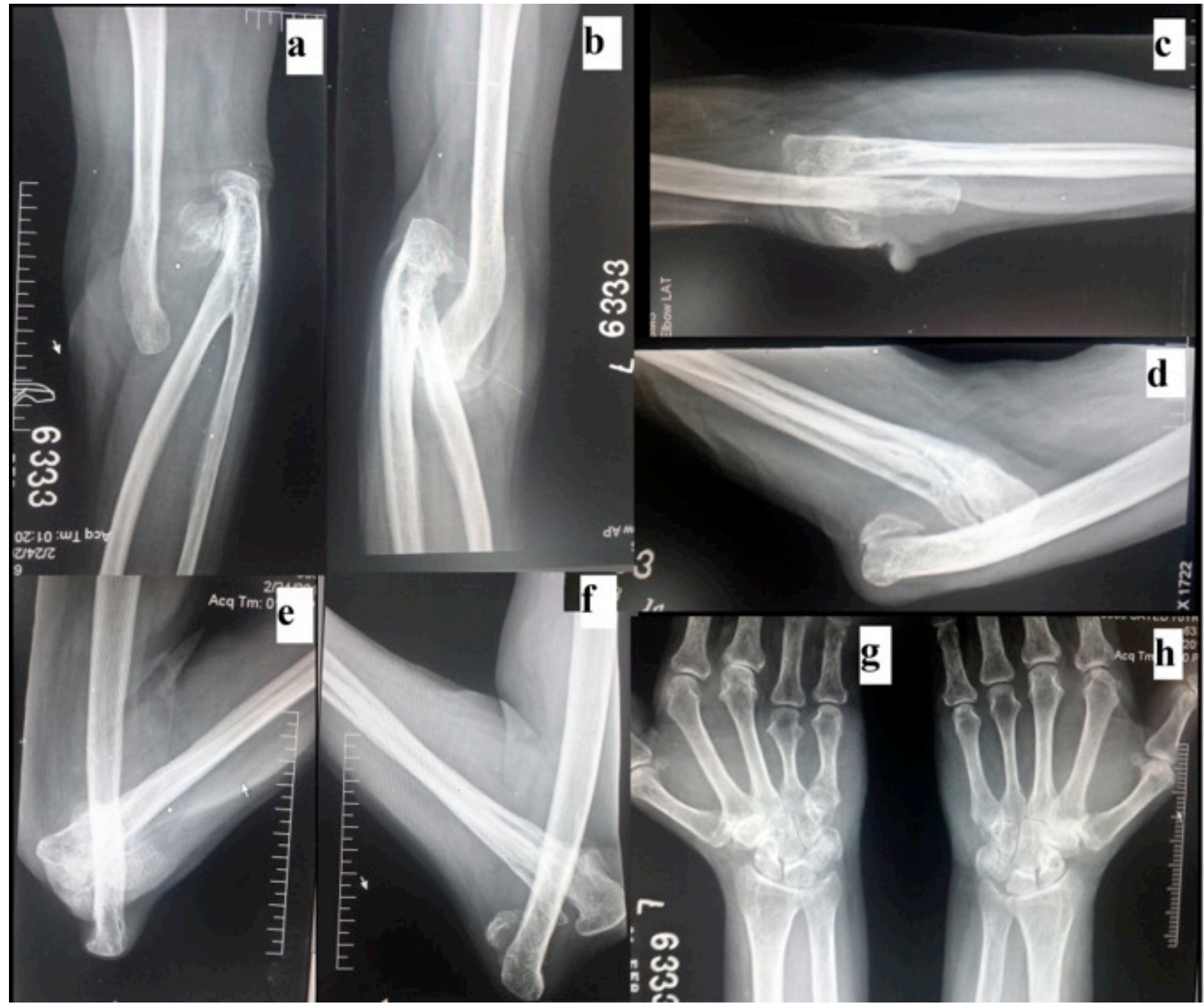

Fig. (4). Radiographs. a, b) Anteroposterior views of bilateral elbow joints showing dislocation of elbow joints and fusion of proximal radius and ulna. Also note heterotopic ossification. The right ulna is hypoplastic. c, d) Lateral views of right and left elbow in maximal possible extension. e, f) Lateral views of right and left elbows in maximal possible flexion. g) Short fourth and fifth metacarpals in the right hand. h) Hypoplasia of the fourth metacarpal in the left hand.

One of the surprising things is that the patient had excellent functionality despite the striking clinico-radiological appearance. The range of motion characteristic of normal elbow and radioulnar joints was preserved to a great extent despite the destruction of the normal anatomy. The patient was a mechanic by occupation and was able to lift heavy weights despite the altered elbow structure and multidirectional instability clinically. Till date, the patient is functionally independent and has no complaints with respect to bilateral elbow joints. This could be partly due to the excellent functional adaptation in children to disabilities as the patient was afflicted at the age of eight years. Cases of osteoarticular sequelae of smallpox may continue to rise occasionally in previously endemic areas till the generation of patients afflicted by smallpox pass on. It is valuable to know the clinico-radiological picture of an already eradicated disease which might avoid unnecessary workup of the patients.

\section{CONCLUSION}

Cases of osteomyelitis variolosa will continue to torment the untrained eyes of surgeons until the generation of patients afflicted by smallpox pass on. It is essential to be aware of the clinical and radiological presentation of the sequelae in the post eradication era to prevent unnecessary investigations. Patients with osteomyelitis variolosa may often continue to have excellent functionality despite the clinico-radiological presentation. This may be partly due to the excellent functional adaptation over the period of time.

\section{CONFLICT OF INTEREST}

The authors confirm that this article content has no conflict of interest.

\section{ACKNOWLEDGEMENTS}

Declared none.

\section{REFERENCES}

[1] Smallpox. Available at: http://www.who.int/mediacentre/factshee ts/smallpox/en/. Accessed December 4, 2006.

[2] Cockshott P, MacGregor M. Osteomyelitis variolosa. Quart J Med 1958; 27: 369-87. 
[3] Cockshott P, MacGregor M. Natural history of osteomyelitis variolosa. J Fac Radiol 1959; 10: 57-63.

[4] Davidson JC, Palmer PES. Osteomyelitis variolosa. J Bone Joint Surg Br 1963; 45B: 687-93.

[5] Klenerman L. A history of osteomyelitis from the Journal of Bone and Joint Surgery 1948 to 2006. J Bone Joint Surg Br 2007; 89B(5): 667-70.

[6] Arora A, Agarwal A, Kumar S. Osteomyelitis variolosa: a report of two cases. J Orthop Surg 2008; 16(3): 355-8.

[7] Balaji D. Osteomyelitis variolosa: a case report. J Orthop Surg (Hong Kong) 2011; 19(1): 120-2.

[8] Lentz MW, Noyes FR. Osseous deformity from osteomyelitis variolosa. A case report. Clin Orthop Relat Res 1979; 143: 155-7.
[9] Nathan PA, Nguyen-Buu-T. Osteomyelitis variolosa: Report of a case. J Bone Joint Surg Am 1974; 56: 1525-8.

[10] Peterson HA. Physeal injury other than fracture. Chapter 3, Infection. Springer 2012; pp. 65-109.

[11] Singh PK. Osteomyelitis variolosa with fracture: a unique case report. Ann Trop Med Public Health 2012; 5: 124-6.

[12] Syed AA, Flanagan JO. Simultaneous bilateral elbow dislocation in an international gymnast. Br J Sports Med 1999; 33: 132-3.

[13] Al Kaissi A, Ganger R, Klaushofer K, Grill F. The management of knee dislocation in a child with Larsen syndrome. Clinics (Sao Paulo). 2011 July; 66(7): 1295-9.

[14] Mohindra Y, Tuli SM. Osteomyelitis variolosa stimulating achondroplasia. Indian J Pediatr 1969; 36: 48-9.

(C) Mugalur et al.; Licensee Bentham Open.

This is an open access article licensed under the terms of the Creative Commons Attribution Non-Commercial License (http://creativecommons.org/licenses/by-nc/3.0/) which permits unrestricted, non-commercial use, distribution and reproduction in any medium, provided the work is properly cited. 